\title{
Cytoreductive Surgery Plus Hyperthermic Intraperitoneal Chemotherapy for Patients with Peritoneal Metastases from Endometrial Cancer
}

\author{
Tommaso Cornali, $\mathrm{MD}^{\mathbf{1}}$, Paolo Sammartino, $\mathrm{MD}, \mathrm{PhD}^{1,2}$, Nikolaos Kopanakis, $\mathrm{MD}, \mathbf{P h D}^{3}$, \\ Athina Christopoulou, $\mathrm{MD}^{4}$, Marialuisa Framarino dei Malatesta, $\mathrm{MD}^{5}$, Elias Efstathiou, $\mathrm{MD}^{3}$, \\ Alessandra Spagnoli, $\mathrm{PhD}^{6}$, Antonio Ciardi, $\mathrm{MD}^{7}$, Daniele Biacchi, MD, $\mathrm{PhD}^{1}$, and John Spiliotis, MD, $\mathrm{PhD}^{3}$ \\ ${ }^{1}$ Department of Surgery "Pietro Valdoni”, Sapienza University of Rome, Rome, Italy; ${ }^{2}$ Department of Surgery "Pietro \\ Valdoni”, Azienda Policlinico Umberto, Rome, Italy; ${ }^{3}$ First Department of Surgical Oncology, Metaxa Cancer Hospital, \\ Pireaus, Greece; ${ }^{4}$ Medical Oncology Unit, S. Andrew Hospital, Patras, Greece; ${ }^{5}$ Department of Gynecology, Obstetric and \\ Urological Science, Oncologic Day-Hospital, Sapienza University of Rome, Rome, Italy; ${ }^{6}$ Department of Public Health \\ and Infection Disease, Statistics Section, Sapienza University of Rome, Rome, Italy; ${ }^{7}$ Department of Radiological \\ Oncological and Pathological Sciences, Sapienza University of Rome, Rome, Italy
}

\begin{abstract}
Background. More information is needed for selection of patients with peritoneal metastases from endometrial cancer (EC) to undergo cytoreductive surgery (CRS) plus hyperthermic intraperitoneal chemotherapy (HIPEC).

Methods. This study analyzed clinical, pathologic, and treatment data for patients with peritoneal metastases from EC who underwent CRS plus HIPEC at two tertiary centers. The outcome measures were morbidity, overall survival (OS), and progression-free survival (PFS) during a median 5 year follow-up period. Uni- and multivariate analyses were performed to identify significant factors related to outcome.

Results. A total of 33 patients met the inclusion criteria and completed the follow-up period. At laparotomy, the median peritoneal cancer index (PCI) was 15 (range 3-35). The CRS procedure required a mean 8.3 surgical procedures per patient, and for 22 patients $(66.6 \%)$, a complete cytoreduction was achieved. The mean hospital stay was 18 days, and major morbidity developed in $21 \%$ of the patients. The operative mortality was $3 \%$. When surgery ended, HIPEC was administered with cisplatin $75 \mathrm{mg} / \mathrm{m}^{2}$ for $60 \mathrm{~min}$ at $43{ }^{\circ} \mathrm{C}$. During a median follow-up period of
\end{abstract}

(C) The Author(s) 2017. This article is an open access publication

First Received: 14 September 2017;

Published Online: 27 December 2017

P. Sammartino, $\mathrm{MD}, \mathrm{PhD}$

e-mail: paolo.sammartino@uniroma1.it
73 months, Kaplan-Meier analysis indicated a 5 year OS of $30 \%$ (median 33.1 months) and a PFS of $15.5 \%$ (median 18 months). Multivariate analysis identified the completeness of cytoreduction (CC) score as the only significant factor independently influencing OS. Logistic regression for the clinicopathologic variables associated with complete cytoreduction $(\mathrm{CC} 0)$ for patients with metachronous peritoneal spread from EC who underwent secondary CRS plus HIPEC identified the PCI as the only outcome predictor.

Conclusions. For selected patients with peritoneal metastases from EC, when CRS leaves no residual disease, CRS plus HIPEC achieves outcomes approaching those for other indications such as colon and ovarian carcinoma.

Endometrial cancer (EC) is the most common gynecologic cancer in developed countries. ${ }^{1}$ For low-risk patients (the majority), primary treatment achieves high overall cancer-specific survival rates, ${ }^{2,3}$ whereas for high-risk patients (20-30\% of all those with a new diagnosis), treatment achieves low survival due to histologically aggressive tumors, adverse pathologic factors, and advanced disease at onset, inducing high recurrent disease rates and accounting for up to $50-60 \%$ of all EC-related deaths. ${ }^{3-6}$

Another common finding (10-30\% overall incidence), especially among patients with recurrent high-risk EC, is intraperitoneal involvement, at a single disease site or combined with hematogenous or lymphatic spread. ${ }^{4,7,8}$ 
Although some reports could underestimate intraperitoneal spread through a transtubal route in early-stage disease, ${ }^{9,10}$ peritoneal disease generally involves patients with primary high-risk advanced-stage EC or those with recurrent tumors, both of whom have a dismal outlook. ${ }^{3,11,12}$

Ongoing trials currently are testing whether a combined approach using cytoreductive surgery (CRS) plus hyperthermic intraperitoneal chemotherapy (HIPEC), already standard care for selected patients with pseudomyxoma peritonei and peritoneal mesothelioma, ${ }^{13}$ improves outcome for peritoneal metastases from colorectal, gastric, and ovarian cancer, as well as for peritoneal surface malignancies (PSM) originating from other unusual sites. ${ }^{14,15}$ Although several retrospective series from centers experienced in treating PSM have analyzed the findings for synchronous or metachronous peritoneal metastases from EC treated with CRS and HIPEC, the small number of patients included preclude statistically reliable general conclusions. $^{16-20}$ Further insights also are needed on prognostic factors and the criteria for selecting patients with peritoneal metastases from EC to undergo CRS and HIPEC.

We therefore investigated a series of patients who had peritoneal metastases from EC treated with CRS plus HIPEC at two tertiary centers experienced in treating PSM. We specifically aimed to assess the results of the integrated procedure achieved in this new PSM field to define outcomes and possible selection criteria more clearly.

The outcome measures were morbidity, overall survival (OS), and progression-free survival (PFS) during a median 5-year follow-up period. Uni- and multivariate analyses were performed to identify significant factors related to outcome.

\section{METHODS}

\section{Study Design}

We conducted a retrospective multicenter cohort study of patients from two tertiary centers experienced in treating PSM. These patients underwent CRS plus HIPEC for peritoneal metastases from EC during the 14 years, from November 2002 to April 2016.

\section{Patient Population}

Data were entered into a custom-designed database including only patients whose records contained complete information including age, Eastern Cooperative Oncology Group (ECOG) performance status, tumor markers, diagnostic techniques, International Federation of Gynecology and Obstetrics (FIGO) stage, ${ }^{21}$ tumor histology, peritoneal cancer index (PCI), ${ }^{22}$ surgical procedures and complications (Clavien-Dindo Classification), ${ }^{23}$ completeness of cytoreduction (CC) score, ${ }^{22}$ HIPEC techniques and drugs, in patients with metachronous peritoneal spread from EC, data regarding primary treatment, chemotherapy, eventual drug-induced toxicity during systemic chemotherapy and HIPEC evaluated with the National Cancer Institute Common Terminology Criteria for Adverse Events (CTCAE version 4.0), ${ }^{24}$ and last, complete, updated data on follow-up.

The indications for CRS and HIPEC were peritoneal metastatic spread from advanced or recurrent EC in patients younger than 75 years with adequate cardiac, renal, hepatic, and bone marrow function and ECOG performance status 0-2 with resectable disease who had signed written informed consent. The contraindications were extra-abdominal disease at CRS plus HIPEC, other malignancies, unresectable disease, and lack of fitness for the procedure. The two institutional review boards approved the study procedures before research activities started and prospective data collection began.

\section{Preoperative Management}

Detailed staging depended chiefly on diagnostic imaging findings including computed tomography (CT), magnetic resonance imaging (MRI), and positron emission CT (PETCT) combined with laparoscopy if imaging failed to specify resectability. Patients were scheduled for CRS plus HIPEC at a multidisciplinary meeting.

\section{CRS and HIPEC}

At laparotomy, peritoneal spread was recorded according to the PCI. ${ }^{22}$ Patients then underwent CRS with peritonectomy procedures and visceral resections intended to leave no visible disease. ${ }^{25}$ For patients who had undergone laparoscopy, trocar sites were removed by fullthickness parietal resection. For patients with synchronous peritoneal spread, CRS included pelvic and paraaortic lymphadenectomy. ${ }^{26}$ Patients with metachronous peritoneal spread from EC underwent lymphadenectomy if it had not been performed previously, or if needed for evident nodal relapse. When surgery ended, HIPEC was administered with the closed technique. ${ }^{27}$

\section{Postoperative Management}

All patients entered an intensive care unit (ICU) to receive prophylaxis for deep venous thrombosis and total parenteral nutrition until oral calorie intake became adequate. Morbidity was analyzed with the Clavien-Dindo 
classification, ${ }^{23}$ and operative mortality was defined as death within 90 days after surgery.

\section{Histopathology and Staging}

Histopathology followed the new FIGO and World Health Organization (WHO) EC classifications including the dualistic classification proposed by Bokhman. ${ }^{28,29}$ All pathology slides underwent central review by a gynecologic pathologist (A.C.).

\section{Follow-up Evaluation}

After CRS plus HIPEC, patients received adjuvant chemotherapy according to their general status and underwent follow-up evaluation according to a predefined scheme standardized for both institutions. For the first 2 years, asymptomatic patients were scheduled for clinical assessment and tumor marker testing every 3 months and diagnostic imaging every 6 months, and thereafter, clinical assessment and tumor marker testing every 6 months and yearly diagnostic imaging. In accordance with Esselen et al. ${ }^{30}$ recurrent disease sites after CRS plus HIPEC were classified as intraperitoneal, extraperitoneal, or distant. Extraperitoneal recurrences were defined as nodal, as were intraabdominal sites outside the peritoneal cavity, including intraparenchymal recurrences. Supradiaphragmatic non-nodal disease was classified as a distant recurrence.

\section{Statistical Analysis}

Follow-up data were completed 31 December 2016. For continuous variables, we analyzed number of observations, median, and range, and for discrete variables, we analyzed number of observations and frequency. The Mann-Whitney $U$ test was used to compare data in groups. In this study, OS was defined as the time from CRS plus HIPEC to the date of death from any cause, and PFS was defined as the time from CRS plus HIPEC to objective tumor progression or death from any cause, whichever occurred first. The study defined PFS2 as the time from CRS plus HIPEC to the second objective disease progression or death from any cause, whichever occurred first. ${ }^{31}$

Both OS and PFS probabilities were estimated using the Kaplan-Meier method. The log-rank test was used to compare between-group OS and PFS. The median OS and PFS and corresponding two-sided $95 \%$ confidence intervals (CIs) for each group were calculated using the KaplanMeier method. Univariate and Cox multivariate regression analysis models were used to explore the influence of prognostic factors on OS and PFS. A logistic regression model was applied to evaluate whether clinicopathologic variables influenced CC scores. Statistical data were analyzed with the $\mathrm{R}$ statistical software package version 3.3.3. All $p$ values lower than 0.05 were considered to indicate statistical significance.

\section{RESULTS}

The inclusion criteria were met by 36 patients attending the two participating tertiary centers who underwent CRS plus HIPEC. Of these 36 patients, 3 were lost to follow-up evalution. Of the remaining 33 patients, 5 had synchronous peritoneal metastases, whereas 28 had metachronous peritoneal metastases from EC and underwent primary or secondary CRS, both combined with HIPEC (Table 1). Three of the five patients for whom laparoscopy confirmed a PCI higher than 20 underwent six cycles of carboplatin AUC6 plus paclitaxel $175 \mathrm{mg} / \mathrm{m}^{2}$ every 3 weeks to lower tumor burden before primary CRS plus HIPEC and had a partial response (intraoperative $\mathrm{PCI}<10$ ). Of the remaining two patients, one refused NeoAdjuvant ChemoTherapy (NACT) and one had a low PCI (3) at diagnosis and directly underwent primary CRS plus HIPEC.

TABLE 1 Demographic and clinical characteristics of the 33 patients with peritoneal metastases from endometrial cancer (EC) undergoing cytoreductive surgery (CRS) plus hyperthermic intraperitoneal chemotherapy (HIPEC)

\begin{tabular}{|c|c|c|}
\hline \multirow[t]{3}{*}{ Variables } & \multicolumn{2}{|c|}{ Peritoneal metastases from EC } \\
\hline & \multicolumn{2}{|l|}{ CRS } \\
\hline & Primary & Secondary \\
\hline No. of patients & 5 & 28 \\
\hline Age: years (range) & $59(42-65)$ & $58(43-73)$ \\
\hline CA-125: U/ml (range) & $150(50-450)$ & $230.5(0-1500)$ \\
\hline \multicolumn{3}{|l|}{ ECOG performance status } \\
\hline 0 & 4 & 12 \\
\hline 1 & 1 & 15 \\
\hline \multicolumn{3}{|l|}{ Histology } \\
\hline \multicolumn{3}{|l|}{ Type 1} \\
\hline \multicolumn{3}{|l|}{ Endometrioid } \\
\hline G2 & 1 & 3 \\
\hline G3 & 1 & 12 \\
\hline \multicolumn{3}{|l|}{ Type 2} \\
\hline Serous & 2 & 8 \\
\hline Clear cell & - & 2 \\
\hline Carcinosarcoma & 1 & 2 \\
\hline Squamous & - & 1 \\
\hline Peritoneal cancer index (range) & $9(3-21)$ & $16(5-35)$ \\
\hline
\end{tabular}

Data are expressed as median and range unless otherwise stated ECOG Eastern Cooperative Oncology Group 
Of the 28 patients who underwent secondary CRS with a median of 17.5 months (range 6-36 months) elapsing after the first operation, 26 had previously undergone total hysterectomy and bilateral salpingo-oophorectomy with locoregional lymphadenectomy (21 pelvic and 5 combined pelvic and paraaortic lymphadenectomy). These 26 patients included 13 who had lymphatic spread at primary treatment and underwent adjuvant chemotherapy using two drugs (cisplatin and doxorubicin) in eight cases, three drugs (cisplatin, doxorubicin, and paclitaxel) in three cases. Two of these patients had a diagnosis of carcinosarcoma using ifosfamide combined with paclitaxel. Finally, four patients underwent vaginal brachytherapy, with two of the four patients receiving combined with adjuvant chemotherapy.

At laparotomy, the median PCI for the 33 patients was 15 (range 3-35), with no significant difference between the patients who underwent primary CRS and those who had secondary CRS ( $p=0.09$, Mann-Whitney $U$ test). Overall, 273 surgical procedures were needed to achieve CRS for the 33 patients. All the patients who underwent primary CRS had pelvic and paraaortic lymphadenectomy, whereas after secondary CRS, 10 patients needed further lymphadenectomy for suspected nodal relapse. Surgery achieved complete cytoreduction (CC score, 0) for 22 patients $(66.6 \%)$, whereas for 11 patients, it left residual disease as follows: CC1 for 7 patients $(21.2 \%), \mathrm{CC} 2$ for 3 patients $(9.1 \%)$, and CC3 for 1 patient $(3.1 \%)$. The mean hospital stay was 18 days, and major morbidity (grade 3 or 4) developed in $21 \%$ of the patients. The overall operative mortality was $3 \%$, involving one patient who had an intraoperative massive pulmonary embolism (Table 2).

For 32 patients (excluding the patient who died intraoperatively of a massive pulmonary embolism), when surgical procedures ended, HIPEC was administered with a single drug (cisplatin $75 \mathrm{mg} / \mathrm{m}^{2}$ ) for $60 \mathrm{~min}$ at $43{ }^{\circ} \mathrm{C}$. For two patients $(6.2 \%)$, HIPEC induced a grade 1 or 2 acute kidney injury, which medical treatment promptly reversed.

At discharge, after a mean hospital stay of 48 days, 30 patients underwent multi-agent adjuvant chemotherapy variously integrated with biologic and molecular treatment for recurrent or metastatic disease. At this writing after a median follow-up period of 73-months (range 8-141 months; 95\% CI 39.05-126.18 months), of the 32 patients who survived after CRS plus HIPEC, 8 are alive and disease free, 5 are alive with disease, and 19 have died.

Of the 33 patients in this study, 24 had recurrent disease (involving the peritoneum in $54.1 \%$ ) and received several chemotherapy regimens or further surgery (Table 3). Kaplan-Meier survival analysis showed a 5-year OS rate of $30 \%$ and a 5 -year PFS rate of $15.5 \%$ (Fig. 1a). The median OS was 33.1 months (PFS 18 months; PFS2 28.2 months). The patients who underwent complete cytoreduction (CCO) had a significantly better OS than the patients whose
TABLE 2 Surgical procedures, outcomes, and morbidity

\begin{tabular}{|c|c|c|c|}
\hline \multirow[t]{2}{*}{ Surgical procedures } & \multicolumn{3}{|c|}{ Cytoreductive surgery } \\
\hline & $\begin{array}{l}\text { Primary } \\
(n=5)\end{array}$ & $\begin{array}{l}\text { Secondary } \\
(n=28)\end{array}$ & $\begin{array}{l}\text { All } \\
\text { patients } \\
(n=33)\end{array}$ \\
\hline \multicolumn{4}{|l|}{ Peritonectomy } \\
\hline Pelvic & 4 & 18 & 22 \\
\hline Subtotal & 1 & 10 & 11 \\
\hline \multicolumn{4}{|l|}{ Visceral resections } \\
\hline \multicolumn{4}{|l|}{ Pelvic } \\
\hline Histero-adnexectomy & 5 & - & 5 \\
\hline Recurrent pelvic mass & - & 20 & 20 \\
\hline Upper vaginectomy & - & 7 & 7 \\
\hline \multicolumn{4}{|l|}{ Gastrointestinal } \\
\hline Left colon & 4 & 13 & 17 \\
\hline Right colon & - & 4 & 4 \\
\hline Right + left colon & - & 1 & 1 \\
\hline Transverse colon & - & - & - \\
\hline Total colectomy & 1 & 1 & 2 \\
\hline Small bowel & - & 19 & 19 \\
\hline Appendectomy & 4 & 14 & 18 \\
\hline Gastric & - & 1 & 1 \\
\hline \multicolumn{4}{|l|}{ Hepatobiliary } \\
\hline Cholecystectomy & 2 & 7 & 9 \\
\hline Atypical hepatic resection & - & 2 & 2 \\
\hline Pancreatic tail & - & 1 & 1 \\
\hline Splenectomy & 1 & 8 & 9 \\
\hline \multicolumn{4}{|l|}{ Genitourinary and others } \\
\hline Partial bladder resection & - & 3 & 3 \\
\hline Nephrectomy & - & 1 & 1 \\
\hline Greater omentectomy & 5 & 28 & 33 \\
\hline Round + falciform ligament & 5 & 28 & 33 \\
\hline $\begin{array}{l}\text { Implant resection/in situ } \\
\text { destruction }\end{array}$ & 1 & 25 & 26 \\
\hline Abdominal wall resection & 5 & 9 & 14 \\
\hline \multicolumn{4}{|l|}{ Lymphadenectomy } \\
\hline Regional & 5 & 10 & 15 \\
\hline Total & 43 & 230 & 273 \\
\hline Mean & 8.6 & 8.2 & 8.3 \\
\hline Outcomes & \multicolumn{2}{|c|}{ Mean } & Range \\
\hline Duration of procedures (min) & \multicolumn{2}{|c|}{375} & $120-660$ \\
\hline Blood loss (ml) & \multicolumn{2}{|l|}{600} & $100-900$ \\
\hline Blood transfusions (U) & \multicolumn{2}{|l|}{3} & $1-7$ \\
\hline Plasma transfusions (U) & \multicolumn{2}{|l|}{4} & $2-8$ \\
\hline ICU stay $(\mathrm{h})^{\mathrm{a}}$ & \multicolumn{2}{|l|}{14} & $8-50$ \\
\hline Postoperative stay (days) & \multicolumn{2}{|c|}{18.6} & $9-90$ \\
\hline Surgical morbidity grade ${ }^{\mathrm{b}}$ & \multicolumn{2}{|l|}{$n$} & $\%$ \\
\hline $1-2$ & \multicolumn{2}{|c|}{10} & 33.3 \\
\hline 3 & 5 & & 15.1 \\
\hline
\end{tabular}


TABLE 2 continued

\begin{tabular}{lll}
\hline Surgical morbidity grade & $n$ & $\%$ \\
\hline 4 & 1 & 3 \\
5 & 1 & 3
\end{tabular}

ICU intensive care unit

${ }^{a}$ hours

${ }^{\mathrm{b}}$ According to Clavien-Dindo classification

surgery left residual disease $(p<0.016, \log$-rank test) (Fig. 1b).

The univariate analysis (log-rank test) for OS identified the CC score and PCI as the only two factors significantly influencing outcome. The univariate analysis for PFS identified the PCI as the only significant factor. The multivariate Cox regression analysis reevaluating significant univariate prognostic factors identified the CC score as the only significant factor capable of independently influencing OS (Table 4). Logistic regression for the clinicopathologic variables associated with complete cytoreduction (CC0) in the 28 patients with metachronous peritoneal spread from EC who underwent secondary CRS combined with HIPEC identified the PCI as the only outcome predictor (OR 1.24; 95\% CI 1.09-1.53). A one-unit increase in PCI value increased the risk of a CC score higher than 0 by $24 \%$.

\section{DISCUSSION}

This series of patients who had peritoneal metastases from EC treated with CRS plus HIPEC at two tertiary centers experienced in treating PSM was relatively large given that peritoneal metastases from EC involve an unusual site for this combined treatment. Despite the rare indications and given that treating these patients is a challenging task, overall, we obtained with acceptable morbidity, outcome rates generally approaching those reported for CRS plus HIPEC in other indications. ${ }^{32-34}$

Our findings in this series of patients with peritoneal metastases from EC treated with CRS plus HIPEC are hard to compare with others. Although two collective reviews report a series of patients who underwent combined treatment for peritoneal metastases from various primary diseases including EC, they failed to analyze the features of these patients in detail. ${ }^{15,33}$ The only homogeneous comparison is in the series of 13 patients treated by Delotte et al. ${ }^{19}$ These investigators reported a complete cytoreduction rate analogous to that in our series, but achieved higher overall survival rates also if the median PCI and median follow-up period were less than the rates we report (median PCI, 12 vs. 15; median follow-up period, 19.4 vs. 73 months).
An equally difficult task was to compare our patients who had advanced or recurrent EC with peritoneal spread treated using CRS plus HIPEC with other patients who underwent CRS alone. In the past few years, therapeutic advances have recommended (e.g., for ovarian cancer) ${ }^{35}$ maximal cytoreduction aimed at leaving no residual disease as the cornerstone of every multimodal therapeutic strategy. ${ }^{36-44}$ Adjuvant chemotherapy unfortunately seems poorly effective for patients with advanced high-risk EC, , $^{3,6}$ leading to considerably lower outcome rates than those obtained for ovarian cancer. ${ }^{45}$ Precise identification of metastatic peritoneal disease and quantification of its extent are factors rarely considered by major case series analyzing the results obtained with CRS alone for primary advanced or recurrent EC. Moreover, when studies refer to peritoneal spread, they usually do so to underscore that this is a typical site for metastatic spread in high-risk patients, worsened by an unfavorable outcome. ${ }^{4,6,8}$ Hence, comparison of outcome findings for our patients treated using CRS plus HIPEC with those for patients who undergo CRS alone suffers from bias. Bias apart, our outcome results compare well with published data, especially for those patients in whom CRS combined with HIPEC achieved complete cytoreduction. ${ }^{12,37,38,41,42,45,46}$

Despite a few exceptions, ${ }^{6,46}$ in our series, compared with most published series analyzing data for CRS alone in treating advanced or recurrent EC, ${ }^{37,38,41,42}$ the only independent prognostic factor able to predict outcome was the completeness of cytoreduction. Although it remains conjectural whether HIPEC improved outcome in our series, an outcome finding that emerged from analysis of the 24 patients who had recurrent disease was the site of recurrence. Only $50 \%$ of the recurrent disease sites involved the peritoneum, thus implying that HIPEC might act as a protective factor. This finding supports what Esselen et al. ${ }^{30}$ have already reported for advanced ovarian cancer treated after CRS with endoperitoneal normothermic adjuvant chemotherapy.

Our series also provided some help in identifying the criteria for selecting CRS plus HIPEC to manage peritoneal metastases from EC. For this purpose, we need to distinguish synchronous from metachronous peritoneal metastases. Despite the few synchronous peritoneal metastases, NACT reduced peritoneal involvement before CRS for three of our five patients, and achieved long-term PFS for the remaining two patients. Given that others have used NACT in primary advanced EC for no more than 100 patients, it still seems premature to consider NACT responses as a selection criterion for CRS with HIPEC. ${ }^{47}$ For the 28 patients with metachronous peritoneal metastases from EC, the logistic regression analysis testing clinicopathologic factors associated with complete cytoreduction identified PCI as the only outcome predictor. A 


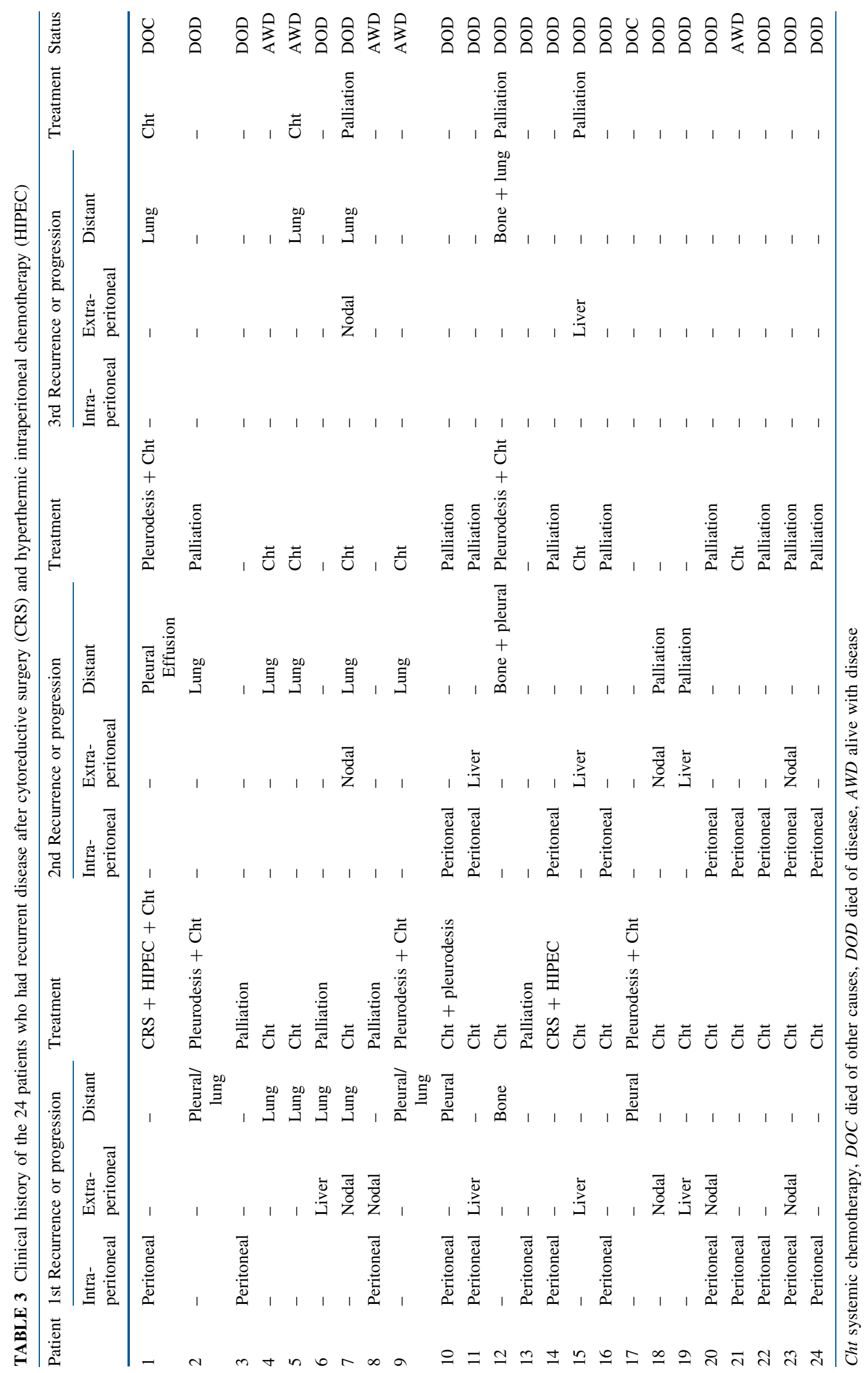




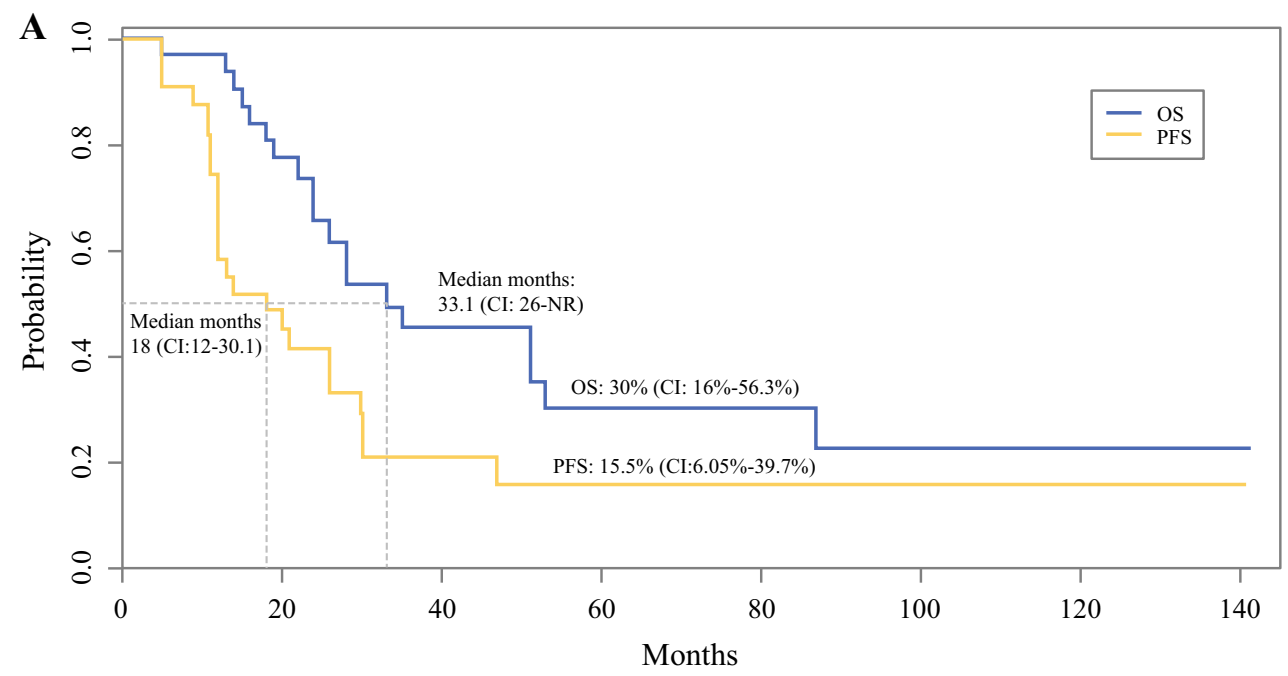

OS by CC-S

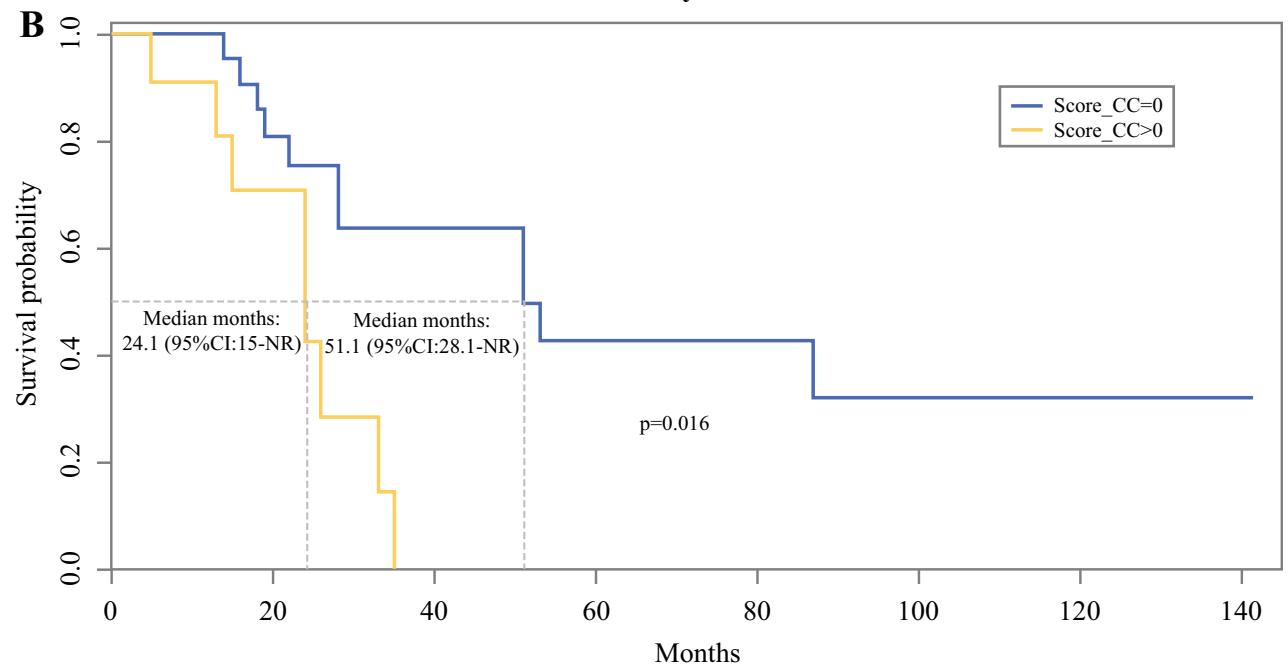

FIG. 1 a Kaplan-Meier curves for overall survival (OS) and progression-free survival (PFS) of the 33 patients with peritoneal metastases from endometrial cancer who underwent cytoreductive surgery (CRS) plus hyperthermic intraperitoneal chemotherapy

possible PCI cutoff value for selecting patients to undergo CRS with HIPEC remains open for future research in a larger cohort.

Because none of our patients with advanced-stage EC had undergone genomic characterization to identify molecular biomarkers predicting individual tumor behavior, we cannot say to which molecular EC subgroup their tumors belonged. ${ }^{48}$ Nor can we say whether molecular status explained the wide survival range. Future research should improve the emerging molecular classification tools for risk group assessment and avoid wasting resources by identifying cost-effective molecular-targeted therapy. ${ }^{49}$
(HIPEC). b Kaplan-Meier curves for overall survival (OS) by the completeness of cytoreduction (CC) score of the 33 patients with peritoneal metastases from endometrial cancer who underwent CRS plus HIPEC

The limitations of our study were its retrospective design and the small number of patients enrolled. During the natural history of EC, only $15-30 \%$ of patients have peritoneal metastatic spread, precluding prospective randomized-controlled trials. ${ }^{39}$

In conclusion, for patients with peritoneal metastases from EC, especially when CRS leaves no residual disease, CRS plus HIPEC administered in experienced centers for properly selected cases achieves OS and PFS outcome rates approaching those for the most frequent indications for this combined procedure (colorectal or ovarian cancer). ${ }^{32,34,50}$ Given that positive peritoneal cytology is a risk factor for peritoneal relapse and that some suggest including 
TABLE 4 Prognostic factors for progression-free survival (PFS) and overall survival (OS) of the patients with peritoneal metastases from evaluable endometrial cancer $(n=32)$ (Cox proportional-hazards model)

\begin{tabular}{|c|c|c|c|}
\hline \multirow[t]{3}{*}{ Variables } & \multicolumn{3}{|l|}{ Survival analysis } \\
\hline & \multicolumn{2}{|l|}{ Univariate } & \multirow{2}{*}{$\begin{array}{l}\text { Multivariate } \\
\text { OS } \\
\text { HR }(95 \% \mathrm{CI})\end{array}$} \\
\hline & $\begin{array}{l}\text { PFS } \\
\text { HR }(95 \% \text { CI })\end{array}$ & $\begin{array}{l}\text { OS } \\
\text { HR }(95 \% \mathrm{CI})\end{array}$ & \\
\hline Age at diagnosis & $1.01(0.97-1.06)$ & $1.02(0.97-1.08)$ & - \\
\hline \multicolumn{4}{|l|}{$\mathrm{CC}$ score } \\
\hline 0 & - & - & - \\
\hline$>0$ & $1.90(0.82-4.41)$ & $3.94(1.39-11.18)$ & $3.92(1.39-11.12)$ \\
\hline \multicolumn{4}{|l|}{ Histology type } \\
\hline 1 & - & - & - \\
\hline 2 & $1.52(0.64-3.43)$ & $1.16(0.47-2.86)$ & \\
\hline PCI & $1.06(1.01-1.12)$ & $1.06(1.01-1.13)$ & - \\
\hline
\end{tabular}

$H R$ hazard ratio, $C I$ confidence interval, $P F S$ progression-free survival, $O S$ overall survival, $C C$ completeness of cytoreduction, $P C I$ peritoneal cancer index (considered as a continuous variable)

omentectomy in staging high-risk EC, ${ }^{9,11,26,51}$ HIPEC plus CRS might help to prevent peritoneal spread in EC.

DISCLOSURE There is no conflicts of interest.

OPEN ACCESS This article is distributed under the terms of the Creative Commons Attribution 4.0 International License (http:// creativecommons.org/licenses/by/4.0/), which permits unrestricted use, distribution, and reproduction in any medium, provided you give appropriate credit to the original author(s) and the source, provide a link to the Creative Commons license, and indicate if changes were made.

\section{REFERENCES}

1. Torre LA, Bray F, Siegel RL, Ferlay J, Lortet-Tieulent J, Jemal A. Global cancer statistics, 2012. CA Cancer J Clin. 2015;65:87-108.

2. Ferlay J, Soerjomataram I, Erwik M, et al. International agency for research on cancer. GLOBOCAN 2012 v1.0. Cancer incidence and mortality worldwide. IARC CancerBase no. 11. http:// globocan.iarc.fr. Accessed 20 Jun 2017.

3. Dowdy SC. Improving oncologic outcomes for women with endometrial cancer: realigning our sights. Gynecol Oncol. 2014;133:370-4.

4. Bendifallah S, Ouldamer L, Lavoue V, et al. Patterns of recurrence and outcomes in surgically treated women with endometrial cancer according to ESMO-ESGO-ESTRO consensus conference risk groups: results from the FRANCOGYN Study Group. Gynecol Oncol. 2017;144:107-12.

5. Bendifallah S, Perrin M, Ouldamer L, et al. Honing the classification of high-risk endometrial cancer with inclusion of lymphovascular space invasion. Surg Oncol. 2017;26:1-7.

6. Ayeni TA, Bakkum-Gamez JN, Mariani A, et al. Comparative outcomes assessment of uterine grade 3 endometrioid, serous and clear cell carcinomas. Gynecol Oncol. 2013;129:478-85.

7. Huang HJ, Tang $\mathrm{YH}$, Chou $\mathrm{HH}$, et al. Treatment failure in endometrial carcinoma. Int J Gynecol Cancer. 2014;24:885-93.
8. Zanfagnin V, Ferrero A, Biglia N, et al. The role of surgery in recurrent endometrial cancer. Expert Rev Anticancer Ther. 2016;16:741-50.

9. Joo WD, Schwartz PE, Rutherford TJ, et al. Microscopic omental metastasis in clinical stage I endometrial cancer: a meta-analysis. Ann Surg Oncol. 2015;22:3695-700.

10. Felix AS, Brinton LA, McMeekin DS, et al. Relationships of tubal ligation to endometrial carcinoma stage and mortality in the NRG Oncology/Gynecologic Oncology Group 210 Trial. J Natl Cancer Inst. 2015. https://doi.org/10.1093/jnci/djv158.

11. Milgrom SA, Kollmeier MA, Abu-Rustum NR, Makker V, Gardner GJ, Barakat RR, Alektiar KM. Positive peritoneal cytology is highly predictive of prognosis and relapse patterns in stage III (FIGO 2009) endometrial cancer. Gynecol Oncol. 2013;130:49-53.

12. Kraemer O, Rapiti E, Huber D, Lopes-Raimundo E, Usel M, Bouchardy C, Petignat P. Stage IVB endometrial cancer: clinical course and survival of patients with single and multiple metastases. Eur J Gynaecol Oncol. 2015;36:529-32.

13. Li Y, Yu Y, Liu Y. Report on the 9(th) International Congress on peritoneal surface malignancies. Cancer Biol Med. 2014;11:281-4.

14. Sugarbaker PH. The seven best from PSOGI 2016. Ann Surg Oncol. 2017;24:870-4.

15. Goéré D, Passot G, Gelli M, Levine EA, Bartlett DL, Sugarbaker $\mathrm{PH}$, Glehen O. Complete cytoreductive surgery plus HIPEC for peritoneal metastases from unusual cancer sites of origin: results from a worldwide analysis issue of the Peritoneal Surface Oncology Group International (PSOGI). Int $J$ Hyperthermia. 2017;33:520-7.

16. Helm CW, Toler CR, Martin RS III, Gordinier ME, Parker LP, Metzinger DS, Edwards RP. Cytoreduction and intraperitoneal heated chemotherapy for the treatment of endometrial carcinoma recurrent within the peritoneal cavity. Int J Gynecol Cancer. 2007;17:204-9.

17. Bakrin N, Cotte E, Sayag-Beaujard A, et al. Cytoreductive surgery with hyperthermic intraperitoneal chemotherapy for the treatment of recurrent endometrial carcinoma confined to the peritoneal cavity. Int J Gynecol Cancer. 2010;20:809-14.

18. Santeufemia DA, Lumachi F, Basso SM, et al. Cytoreductive surgery with hyperthermic intraperitoneal chemotherapy as 
salvage treatment for a late wound recurrence of endometrial cancer. Anticancer Res. 2013;33:1041-4.

19. Delotte J, Desantis M, Frigenza M, Quaranta D, Bongain A, Benchimol D, Bereder JM. Cytoreductive surgery with hyperthermic intraperitoneal chemotherapy for the treatment of endometrial cancer with peritoneal carcinomatosis. Eur J Obstet Gynecol Reprod Biol. 2014;172:111-4.

20. Abu-Zaid A, Azzam AZ, AlOmar O, Salem H, Amin T, AlBadawi IA. Cytoreductive surgery and hyperthermic intraperitoneal chemotherapy for managing peritoneal carcinomatosis from endometrial carcinoma: a single-center experience of 6 cases. Ann Saudi Med. 2014;34:159-66.

21. Mutch DG, Prat J. 2014 FIGO staging for ovarian, fallopian tube, and peritoneal cancer. Gynecol Oncol. 2014;133:401-4.

22. Jacquet P, Sugarbaker PH. Clinical research methodologies in diagnosis and staging of patients with peritoneal carcinomatosis. Cancer Treat Res. 1996;82:359-74.

23. Dindo D, Demartines N, Clavien PA. Classification of surgical complications: a new proposal with evaluation in a cohort of 6336 patients and results of a survey. Ann Surg. 2004;240:205-13.

24. Common terminology criteria for adverse events (CTCAE) Version 4.0. National Cancer Institute, 2009.

25. Sugarbaker PH. Cytoreductive surgery and perioperative chemotherapy for peritoneal surface malignancy: textbook and video atlas. Cine-Med Publishing Inc., Woodbury; 2013.

26. Frederick PJ, Straughn JM Jr. The role of comprehensive surgical staging in patients with endometrial cancer. Cancer Control. 2009;16:23-9.

27. Di Giorgio A, Naticchioni E, Biacchi D, et al. Cytoreductive surgery (peritonectomy procedures) combined with hyperthermic intraperitoneal chemotherapy (HIPEC) in the treatment of diffuse peritoneal carcinomatosis from ovarian cancer. Cancer. 2008;113:315-25.

28. Amant F, Mirza MR, Creutzberg CL. Cancer of the corpus uteri. Int J Gynaecol Obstet. 2012;119(Suppl 2):S110-7.

29. Jones NL, Xiu J, Chatterjee-Paer S, et al. Distinct molecular landscapes between endometrioid and nonendometrioid uterine carcinomas. Int J Cancer. 2017;140:1396-404.

30. Esselen KM, Rodriguez N, Growdon W, Krasner C, Horowitz NS, Campos S. Patterns of recurrence in advanced epithelial ovarian, fallopian tube, and peritoneal cancers treated with intraperitoneal chemotherapy. Gynecol Oncol. 2012;127:51-4.

31. European Medicines Agency. Guideline on the evaluation of anticancer medical products in man (13 December 2012). http:// www.ema.europa.eu/docs/en_GB/document_library/Scientific_ guideline/2013/01/WC500137128.pdf. Accessed 31 Oct 2014.

32. Elias D, Gilly F, Boutitie F, et al. Peritoneal colorectal carcinomatosis treated with surgery and perioperative intraperitoneal chemotherapy: retrospective analysis of 523 patients from a multicentric French study. J Clin Oncol. 2010;28:63-8.

33. Passot G, Vaudoyer D, Villeneuve L, et al. What made hyperthermic intraperitoneal chemotherapy an effective curative treatment for peritoneal surface malignancy: a 25-year experience with 1125 procedures. J Surg Oncol. 2016;113:796-803.

34. Ihemelandu C, Sugarbaker PH. Management for peritoneal metastasis of colonic origin: role of cytoreductive surgery and perioperative intraperitoneal chemotherapy: a single institution's experience during two decades. Ann Surg Oncol. 2017;24:898-905.
35. Bristow RE, Tomacruz RS, Armstrong DK, Trimble EL, Montz FJ. Survival effect of maximal cytoreductive surgery for advanced ovarian carcinoma during the platinum era: a metaanalysis. J Clin Oncol. 2002;20:1248-59.

36. Barlin JN, Puri I, Bristow RE. Cytoreductive surgery for advanced or recurrent endometrial cancer: a meta-analysis. Gynecol Oncol. 2010;118:14-8.

37. Shih KK, Yun E, Gardner GJ, Barakat RR, Chi DS, Leitao MM Jr. Surgical cytoreduction in stage IV endometrioid endometrial carcinoma. Gynecol Oncol. 2011;122:608-11.

38. Ren Y, Shan B, Shi D, Wang H. Salvage cytoreductive surgery for patients with recurrent endometrial cancer: a retrospective study. BMC Cancer. 2014;14:135.

39. Burke WM, Orr J, Leitao M, et al. Endometrial cancer: a review and current management strategies: part I. Gynecol Oncol. 2014;134:385-92.

40. Burke WM, Orr J, Leitao M, et al. Endometrial cancer: a review and current management strategies: part II. Gynecol Oncol. 2014;134:393-402.

41. Alagkiozidis I, Grossman A, Tang NZ, et al. Survival impact of cytoreduction to microscopic disease for advanced-stage cancer of the uterine corpus: a retrospective cohort study. Int J Surg. 2015;14:61-6.

42. Papadia A, Bellati F, Ditto A, et al. Surgical treatment of recurrent endometrial cancer: time for a paradigm shift. Ann Surg Oncol. 2015;22:4204-10.

43. Colombo N, Creutzberg C, Amant F, et al. ESMO-ESGO-ESTRO consensus conference on endometrial cancer: diagnosis, treatment and follow-up. Int J Gynecol Cancer. 2016;26:2-30.

44. Bernardini MQ, Gien LT, Lau S, et al. Treatment-related outcomes in high-risk endometrial carcinoma: Canadian high-risk endometrial cancer consortium (CHREC). Gynecol Oncol. 2016;141:148-54.

45. Landrum LM, Moore KN, Myers TK, Lanneau GS Jr, McMeekin DS, Walker JL, Gold MA. Stage IVB endometrial cancer: does applying an ovarian cancer treatment paradigm result in similar outcomes? A case-control analysis. Gynecol Oncol. 2009;112:337-41.

46. Holman LL, Pal N, Iglesias DA, et al. Factors prognostic of survival in advanced-stage uterine serous carcinoma. Gynecol Oncol. 2017;146:27-33.

47. Rabinovich A. Neo-adjuvant chemotherapy for advanced-stage endometrial carcinoma: a glimmer of hope in select patients. Arch Gynecol Obstet. 2016;293:47-53.

48. Stelloo E, Nout RA, Osse EM, et al. Improved risk assessment by integrating molecular and clinicopathological factors in earlystage endometrial cancer-combined analysis of the PORTEC Cohorts. Clin Cancer Res. 2016;22:4215-24.

49. Kunitomi H, Banno K, Yanokura $M$, et al. New use of microsatellite instability analysis in endometrial cancer. Oncol Lett. 2017;14:3297-301.

50. Di Giorgio A, De Iaco P, De Simone M, et al. Cytoreduction (peritonectomy procedures) combined with hyperthermic intraperitoneal chemotherapy (HIPEC) in advanced ovarian cancer: retrospective italian multicenter observational study of 511 cases. Ann Surg Oncol. 2017;24:914-22.

51. Altman AD, Ferguson SE, Atenafu EG, et al. Canadian high-risk endometrial cancer (CHREC) consortium: analyzing the clinical behavior of high risk endometrial cancers. Gynecol Oncol. 2015;139:268-74. 